\title{
Preparation of carbon nanofibers by the combination of electrospinning and thermal treatment for electrochemical detection of dopamine, ascorbic acid and uric acid
}

\author{
Chunyan Wang ${ }^{1,2, a}$, Xiaoqiu $\mathrm{Liu}^{1}$ and Xiuhua $\mathrm{Wu}^{1}$ \\ ${ }^{1}$ School of Food Engineering, Jinlin Engineering Normal University, Changchun, Jilin 130052, \\ China \\ ${ }^{2}$ State Key Laboratory of Electroanalytical Chemistry, Changchun Institute of Applied Chemistry, \\ Chinese Academy of Sciences, Changchun, Jilin 130022, China \\ wangcy@ciac.ac.cn
}

\begin{abstract}
Keywords: carbon nanofibers, electrospinning, thermal treatment, detection, dopamine, ascorbic acid, uric acid

Abstract Carbon nanofibers (CNFs) was synthesized by simple electrospinning, followed by thermal treatment over a carbonization temperature of $900{ }^{\circ} \mathrm{C}$. The morphology and microstructure of CNFs were characterized by scanning electron microscopy (SEM) and transmission electron microscopy (TEM). The results indicate that CNFs exhibited small diameter and rough surface, could be directly used for the electrode modification. The prepared CNFs showed high catalytic activity toward the oxidation of dopamine (DA), ascorbic acid (AA) and uric acid (UA) and could be used for the selective determination of DA, AA and UA with a low detection limit. With the good selectively and sensitivity, the present method was applied to the determination of DA in injection and UA in urine samples.
\end{abstract}

\section{Introduction}

As a novel and promising carbon nanomaterials, carbon nanofibers (CNFs) shows wide applications in electrocatalysis and chem/biosensing due to their unique nanostructure, high mechanical strength, excellent electrical conductivity and good stability. In the recent years electrospinning has been widely used to produce various polymer nanofibers and was thought to be a highly versatile fiber fabrication method. Electrospining technique uses the electrostatic force to produce continuous fibers with diameters ranging from a few nanometers to several micrometers from a polymeric solution. People prepared CNF and its composites, such as various metal nanoparticle-loaded CNF based on electrospinning technique $[1,2]$.

Dopamine (DA), ascorbic acid (AA) and uric acid (UA) are compounds of great biomedical interest, playing significant roles in the functioning of the human metabolism, central nervous and renal systems [3]. Thus, the determination of DA, AA and UA is important. Because of highly electrochemical activity of DA, AAand UA, electrochemical methods have been widely utilized for their determination [4].

In this work, we develop a simple and controllable approach to prepare CNFs by combination of electrospinning technique with subsequent thermal treatment. The as-obtained CNFs exhibited rough surface, small diameter and CNFs film could be directly used for the electrode modification. When applied to electrochemistry, the electrospun CNF without any pretreatment exhibited large electroactive area and facile electron-transfer kinetics. The resulting CNFs showed good catalytic activity toward the oxidation of DA, AA and UA. A glass carbon electrode (GCE) modified by CNFs film could be used for the selective determination of DA, AA and UA with a low detection limit. The proposed method was applied to determination of DA and UA in real samples with satisfactory results.

\section{Experimental}

Chemical and Solutions. All the reagents were of analytical grade and used without further purification. Polyacrylonitrile $(\mathrm{PAN}, \mathrm{Mw}=150,000)$ and chitosan solution $(5 \mathrm{wt} \%)$ were purchased 
from Sigma-Aldrich Co. (USA). N, N-Dimethylformamide (DMF) were obtained from Beijing chemical Co. (China). Dopamine (DA), ascorbic acid (AA) and uric acid (UA) were obtained from Beijing Dingguo Biotechnology Co., Ltd. (China). The double distilled water was used for solution preparation. Phosphate buffer solutions (PBS, $0.1 \mathrm{M}$ ) of different $\mathrm{pH}$ were prepared from stock solutions of $0.1 \mathrm{M} \mathrm{H}_{3} \mathrm{PO}_{4}, \mathrm{NaH}_{2} \mathrm{PO}_{4}, \mathrm{Na}_{2} \mathrm{HPO}_{4}$ and $\mathrm{NaOH}$.

Apparatus. The scanning electron microscopy (SEM) experiment was carried out using a PHILIPS XL-30 ESEM at an accelerating voltage of $20 \mathrm{kV}$. The transmission electron microscopy (TEM) images were acquired with a TECNAI G2 with the accelerating voltage of $200 \mathrm{kV}$. Cyclic voltammetry (CV) and differential pulse voltammetry (DPV) were performed with a CHI 660 electrochemical analyzer (USA). A three-electrode system was used, where a GCE $(\mathrm{d}=3 \mathrm{~mm})$ modified with CNFs film was used as the working electrode, a platinum wire as the counter electrode and a $\mathrm{Ag} / \mathrm{AgCl}(3 \mathrm{M}$ $\mathrm{KCl})$ electrode as the reference electrode.

Synthesis of CNFs [5]. The CNFs were synthesized by carbonizing the PAN fibers, which were made via an electrospinning technique by using homogeneous $10 \%$ wt. PAN solution in DMF. During the electrospinning process, the voltage was kept as $18 \mathrm{kV}$, the distance between the spinneret and the collector was kept as $14 \mathrm{~cm}$ and the injection rate is $1 \mathrm{~mL} \mathrm{~h}^{-1}$. For stabilization and carbonization of PAN fibers, they were completed in a high temperature furnace by the following steps: 1) $300^{\circ} \mathrm{C}$ annealing in air for $1 \mathrm{~h}$ to oxidize PAN partially; 2) heating up to $900^{\circ} \mathrm{C}$ under the heating rate of $10^{\circ} \mathrm{C}$ $\min ^{-1}$ and stayed at this temperature for $1 \mathrm{~h}$ for the shape stabilization and combination of the fibers, and then cooled to room temperature in Ar atmosphere.

Preparation of modified electrode. Prior to modification, GCE was polished with alumina slurry $(0.3$ $\mu \mathrm{m}$ and $0.05 \mu \mathrm{m})$ on a chamois leather, and then washed and ultrasonically cleaned in ethanol and redistilled water, respectively. Finally, the clean GCE was dried with $\mathrm{N}_{2}$. The CNFs film was well cut out and then adhibited onto the clean GCE surface with $2 \mu \mathrm{L}$ of $0.5 \mathrm{wt} \%$ chitosan solution. This modify method was convenient and effective compared that CNFs were dissolved and dispersed to modify electrode.

\section{Results and discussion}

Synthesis and characteristics of CNFs. Electrospinning is an efficient and versatile approach for the preparation of long nanofibers, and subsequent carbonization of the electrospun nanofibers at high temperature could produce CNFs [1]. Herein, the PAN nanofibers were firstly synthesized based on electrospinning technique as the precursor of CNFs. Then PAN nanofibers were stabilized and carbonized to produce the CNFs without any catalyst involved during this process. So the pure CNFs could be obtained and used directly without any oxidative pretreatment.
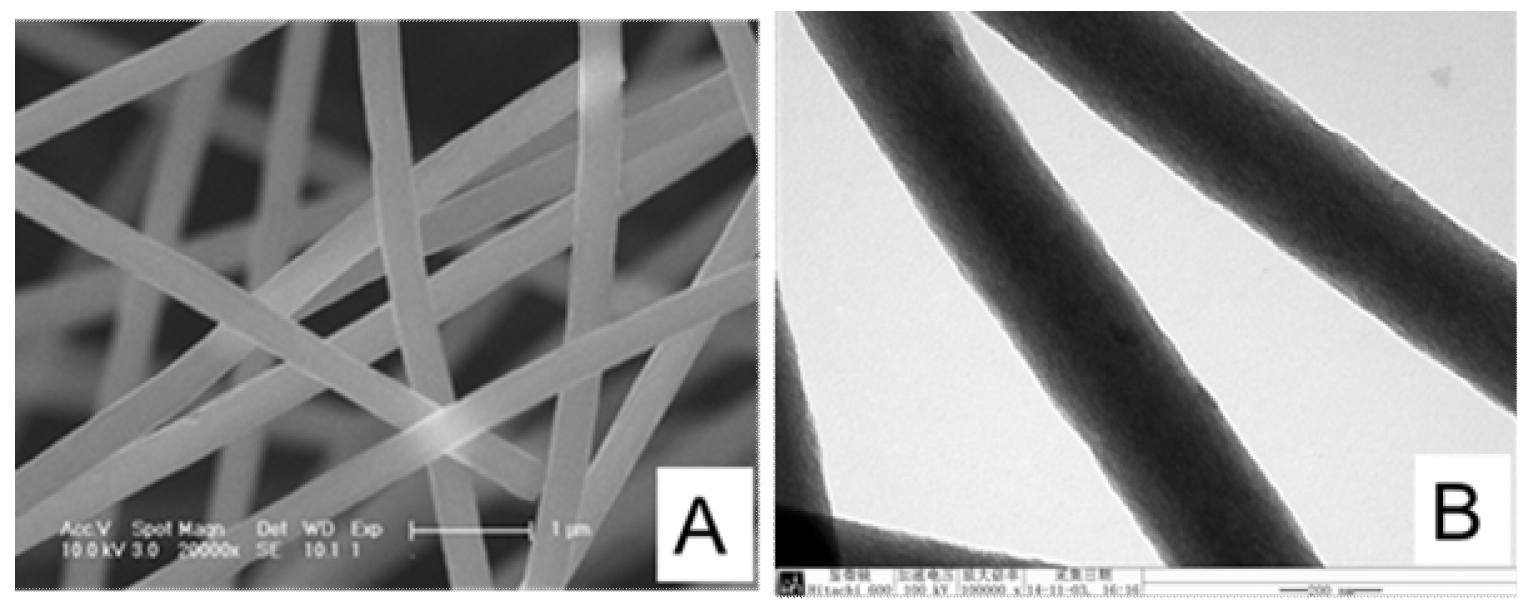

Fig. 1. (A) SEM and (B) TEM image of the CNFs. 
At first, SEM was performed to characterize the surface morphology of CNFs. As shown in Fig. 1A, the CNFs with a diameter ranging from $200 \mathrm{~nm}$ to $400 \mathrm{~nm}$ and tens of micrometers in length are loosely interwaved together and form a network-like structure. This three-dimensional porous film structure is greatly beneficial in maintaining large electroactive area on the electrode surface and facilitating the diffusion of analytes when applied to electrochemial reaction. Fig. 1B shows the typical TEM image of the as-prepared CNFs, further showing a rough and porous structure. This increased significantly the electroactive area of the CNFs.

Electrochemical detection of DA, AA and UA. Fig. 2 compares the voltammetric responses of different electrodes towards single determination of DA, AA and UA. At GCE (Fig. 2A), the oxidation peaks potential of DA, AA and UA are observed at $410 \mathrm{mV}, 344 \mathrm{mV}$ and $600 \mathrm{mV}$, respectively. In addition, DA, AA and UA demonstrate a broad oxidation peak, so that the separation of these three species is not large enough to obtain good selectivity at the bare GCE. While at the CNFs-GCE (Fig. 2B), the oxidation peak potential of DA, AA and UA are observed at $220 \mathrm{mV},-200 \mathrm{mV}$ and $340 \mathrm{mV}$, respectively, indicating the negative shift to some extent. At the same time, the good separation of DA, AA and UA was enlarged. In addition, the peak current of DA, AA and UA showed a notable increase compare with that at the GCE. The results display that the CNFs-GCE exhibited good electrocatalytic activities towards the oxidation of DA, AA and UA. This behavior mainly attributed to the good conductivity of CNFs and the three-dimensional network-like structure of CNFs which improve the electroactive area and facilitate the accumulation of the species. Simultaneously, a large number of defective sites on CNFs would be beneficial for accelerating electron-transfer between the electrode and species in solution $[6,7]$.
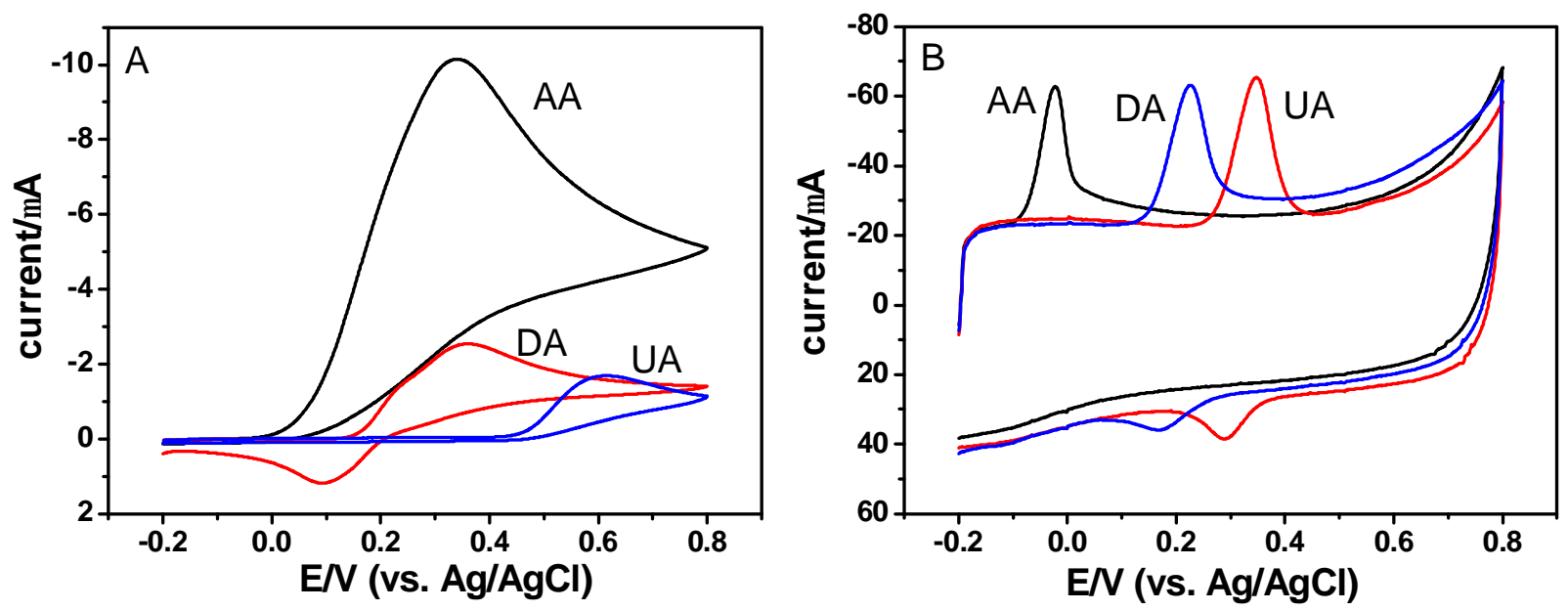

Fig. 2. CVs at (A) GCE and (B) CNF-GCE in $0.1 \mathrm{M}$ PBS (pH 7.0) containing 2.0 mM AA, $0.2 \mathrm{mM}$ DA and $0.3 \mathrm{mM} \mathrm{UA}$, respectively. Scan rate: $50 \mathrm{mV} \mathrm{s}^{-1}$.

The enlarged separation of the oxidation peak potential, coupled with the increased sensitivity, make it possible to effectively determine DA, AA and UA simultaneously. As shown in Fig. 3A, the voltammetric response of GCE toward the mixture of DA, AA and UA is showed as a broad and weak peak (curve a), indicating the poor selectivity and sensitivity. In contrast, at the CNFs-GCE, three well-defined peaks with remarkably improved peak current can be observed (curve b), indicating the simultaneous determination of DA, AA and UA could be obtained at the CNF-GCE.

DPV is a sensitive technique which has been usually undertaken for detection of DA, AA and UA. We have also applied to determine DA, AA and UA at the GCE and CNFs-GCE. Fig. 3B shows the DPV of the mixture solution of DA, AA and UA at the bare GCE and CNFs-GCE. It seems that the separation of the oxidation peak potentials for DA, AA and UA is not enough to obtain good selectivity at the bare GCE (curve a). While DPV peaks for the oxidation of DA, AA and UA in their mixture are well-resolved with significantly improved peak current at the CNFs-GCE, which is enough to determine DA, AA and UA individually or simultaneously. 

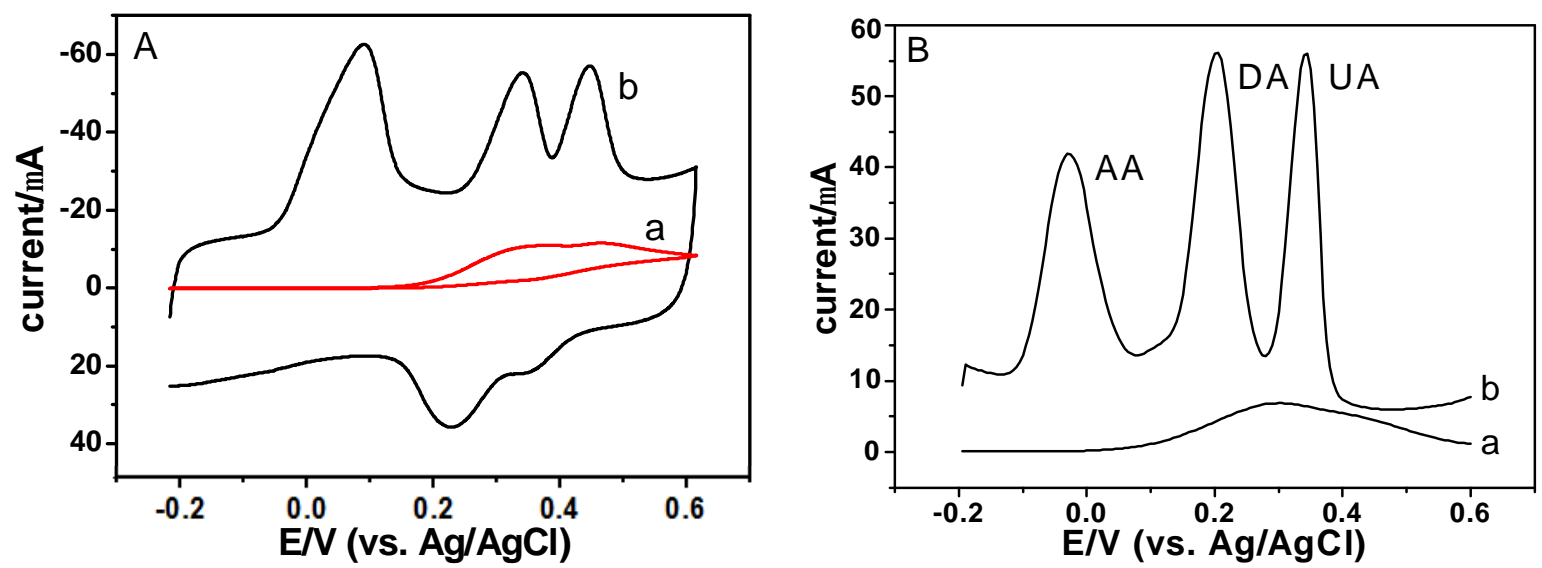

Fig. 3. CVs (A) and DPVs (B) at GCE (curve a) and CNF-GCE (curve b) in 0.1 M PBS (pH 7.0) mixing $2.0 \mathrm{mM}$ AA, $0.2 \mathrm{mM}$ DA and $0.3 \mathrm{mM}$ UA. Scan rate: $50 \mathrm{mV} \mathrm{s}^{-1}$.

DPV was used to individual detection of DA, AA and UA at the CNFs-GCE. The linear range for DA, AA and UA were $0.5-100 \mu \mathrm{M}, 2-400 \mu \mathrm{M}$ and $1-100 \mu \mathrm{M}$ with detection limits of $0.3 \mu \mathrm{M}, 1.0 \mu \mathrm{M}$ and $0.5 \mu \mathrm{M}(\mathrm{S} / \mathrm{N}=3)$, respectively. Additionally, We further studied the simultaneous and quantitive determination of DA, AA and UA at the CNFs-GCE. The concentration of one species changed with the other two keeping constant during the measurement. The results showed that the peak current of DA, AA or UA is proportional to its concentration while the peak current of other two compounds almost keeps constant, so that the oxidation of DA, AA and UA at the CNFs-GCE are relatively independent. The CNFs-GCE shows good selectivity, sensitivity and stability, making it attractive as a sensor for simultaneous detection of DA, AA and UA in biological fluids.

Samples analysis. To investigate the practical applicability of the proposed method for the determinations of DA, AA and UA, dopamine hydrochloride injection (10 $\mathrm{mg} \mathrm{mL}^{-1}, 2 \mathrm{~mL}$ per injection) and human urine samples were tested. The dopamine hydrochloride injection was diluted 10 times with double distilled water. All the urine samples were diluted 100 times with PBS (pH 7.0). No other pretreatment process was performed before the measurement. And then appropriate amounts of the diluted sample were transferred into the electrochemical cell for the determination of DA and UA at the CNFs-GCE using DPV. The standard addition method was used for testing recovery. The recovery rates of the spiked samples ranged from $96.2 \%$ to $105.1 \%$ and $95.6 \%$ to $102.3 \%$ for DA and UA, respectively. The results are acceptable, indicating that the CNFs-GCE could be effectively used in the real samples and free from interferences of the sample matrix.

\section{Conclusions}

CNFs were prepared by electrospinning and subsequent thermal treatment processes. CNFs-GCE displayed excellent electrochemical catalytic activities towards DA, AA and UA. The calibration curves were obtained in the range of $0.5-100 \mu \mathrm{M}, 2-400 \mu \mathrm{M}$ and 1-100 $\mu \mathrm{M}$ and the lowest detection limits were $0.3 \mu \mathrm{M}, 1.0 \mu \mathrm{M}$ and $0.5 \mu \mathrm{M}$ for DA, AA and UA, respectively. The CNFs-GCE could be applied effectively to the detection of DA in injectable medicine and UA in urine sample with satisfying results. And promisingly, the CNF is a candidate for electroanalytic application.

\section{References}

[1] H. Hou, D. Reneker. Adv. Mater. 16 (2004), p.69-73.

[2] J. Huang, D. Wang, H. Hou, et al. Adv. Funct. Mater. 18 (2008), p.441-448.

[3] S. Yang, G. Li, R. Yang, et al. J. Solid State Electrochem. 15 (2011), p.1909-1918.

[4] R. McCreery. Chem. Rev. 108 (2008), p.2646-2687.

[5] Y. Liu, H. Hou, T. You. Electroanalysis 20 (2008), p.1708-1713.

[6] C. Banks, R. Compton. Analyst 131 (2006), p.15-21. 
[7] M. Zhou, Y. Zhai, S. Dong. Anal. Chem. 81 (2009), p.5603-5613. 